\title{
Joint Discussion 12 Long wavelength astrophysics
}

\author{
T. Joseph W. Lazio and Namir E. Kassim (eds.) \\ Naval Research Laboratory, 4555 Overlook Ave. SW, Washington, DC 20375, USA \\ email: joseph.lazio,namir.kassim@nrl.navy.mil
}

\section{Preface}

The greatest discoveries in astronomy have accompanied technological innovations that have opened new windows of the electromagnetic spectrum. One of the last poorly explored regions lies at wavelengths longer than $3 \mathrm{~m}(100 \mathrm{MHz})$ to the ionospheric cutoff around $30 \mathrm{~m}(10 \mathrm{MHz})$. In the past, variations in the ionosphere have limited groundbased instruments to small $(<5 \mathrm{~km})$ apertures and hence relatively coarse angular resolution and low sensitivity.

Ever-increasing computing power combined with new wide-scale imaging algorithms, self-calibration techniques, and angle-dependent calibration schemes make it possible to overcome this restriction. With these advantages, the Very Large Array's $74 \mathrm{MHz}(4 \mathrm{~m})$ observing system and the Indian GMRT have generated many exciting scientific results in the past several years and elegantly demonstrate that connected element interferometry at long wavelengths is no longer limited by phase fluctuations from the ionosphere

With the world-wide activitiy - both the scientific results being generated at the existing facilities and the new facilities that are coming on-line shortly - the IAU XXVI General Assembly seemed timely. Joint Discussion 12 on Long Wavelength Astrophysics, was a one-day session at the General Assembly organized to review the current results as well as anticipate those to come shortly.

Reception to JD 12 was enthusiastic, clearly demonstrating the renaissance in this area. A total of 20 oral and 35 poster presentations were made, with scientific topics ranging from the nearby (the Earth's ionosphere) to the most distant (the Universe itself at the epoch of reionization). Nearly a third of the oral presentations described instruments either under development or in construction indicating that the future of long wavelength astrophysics appears quite bright.

We thank all of the presenters for their exciting presentations, a sampling of which is presented here. We also thank our fellow members of the SOC for their excellent suggestions and guidance, and K. Kellermann who assisted at the last minute.

\section{Scientific Organizing Committee}

Franklin Briggs (Australia), Gloria Dubner (Argentina), Luigina Feretti (Italy), Nasir E. Kassim (USA, co-chair), T. Joseph Lazio (USA, co-chair), A.V. Megn (Ukraine), Alexander A. Konovalenko (Ukraine), Alain Lecacheux (France), A. Pramesh Rao (India), Huub J.A. Röttgering (Netherlands), and Kurt W. Weiler (USA).

\section{Acknowledgements}

Basic research in radio astronomy at the NRL is supported by 6.1 Base funding.

Joseph Lazio and Nasir Kassim,

Washington DC, November 2006 